Journal of Computer Science 3 (6): 441-448, 2007

ISSN 1549-3636

(C) 2007 Science Publications

\title{
Simulation of the Enhanced Associativity Based Routing Protocol for Mobile Ad Hoc Networks (MANET)
}

\author{
${ }^{1}$ Ayman Mansour Murad and ${ }^{2}$ Bassam Al-Mahadeen \\ ${ }^{1}$ Education Science Faculty - UNRWA, Jordan, P.O Box 140157 Amman Jordan \\ ${ }^{2}$ Department of Mathematics and Computer, Tafilah Technical University, Jordan
}

\begin{abstract}
The simulations results showed that the overall EABR enhancement, when compared with $\mathrm{ABR}$, in terms of communication overhead was ranging from $36 \%$ to $56 \%$. While the overall EABR enhancement in terms of number of operation required to reconstructing the route was ranging from $36 \%$ to $55 \%$. These enhancements were contributed to the novel way in route reconstructing introduced by EABR.
\end{abstract}

Keywords: ad-hoc mobile Network, ABR, EABR, Associativity, Simulation

\section{INTRODUCTION}

In ${ }^{[2]}$ a new method for route reconstruction for the $\mathrm{ABR}^{[1]}$ was proposed. The routing table was amended with a Serial No field to enhance the optimization while reconstruction of the route. Three $\mathrm{MH}$ movements were covered, namely SRC, IN and DEST.

The route re-construction process makes use of the advantage of locality of neighbouring Mobile Hosts (MHs) to quickly construct alternate and even shorter routes, i.e. route optimization through using the Here I am packets.

In the original ABR the DEST role in route reconstruction was passive in the case of DEST movement, while in EABR the DEST has an active role in route reconstruction. Beside that, ABR route invalidation was always performed toward the DEST in the case of IN movement, while an optimization was achieved in EABR to invalidate the shortest partial route from the IN toward either the DEST or the SRC.

In ${ }^{[3]}$ the communication and operation complexity analysis for two protocols namely the ABR and the EABR was presented. The operation complexity and communication complexity as defined in ${ }^{[1]}$ were compared for both the ABR and the EABR, where the values represent the worst-case analysis.

The EABR proofed to be better in route reconstruction, which is attributed to the novel way in which the EABR reconstruct the route after movement of any node and the active role of the moved node in route reconstruction phase ${ }^{[3]}$.

\section{MATERIALS AND METHODS}

Study of Communication Overhead:

Network $=30-50$ Nodes - Route $=30$ nodes

In this scenario the number of nodes, which constitute the route, are 30 while the number of nodes constitute the entire network are varying from 30 to 50 . Different results were observed as shown in Figs. 1, 2, \& 3 .

The simulation results showed that the EABR enhancement in route reconstruction ranges from $36 \%$ to $41 \%$ under different topologies. In cases when the reconstruction is done in the first half of the route the enhancement ranges from $60 \%$ to $70 \%$ under the different topologies, while enhancement reached $11 \%$ when the reconstruction is done in the second half of the route. The communication statistics are summarized in Table 1 .

Table 1: Communication Statistics - Route $=30$ nodes under different topologies

\begin{tabular}{lccc}
\hline Number of Nodes in Network & 50 & 40 & 30 \\
$\begin{array}{l}\text { EABR Enhancement in Communication } \\
\text { EABR Enhancement in Communication in case }\end{array}$ & $41 \%$ & $38 \%$ & $36 \%$ \\
$\begin{array}{l}\text { EA reconstruction point fail in the 1st half of } \\
\text { the route }\end{array}$ & $70 \%$ & $66 \%$ & $60 \%$ \\
$\begin{array}{l}\text { EABR Enhancement in Communication in case } \\
\text { the reconstruction point fail in the 2nd half of } \\
\text { the route }\end{array}$ & & \\
\hline
\end{tabular}

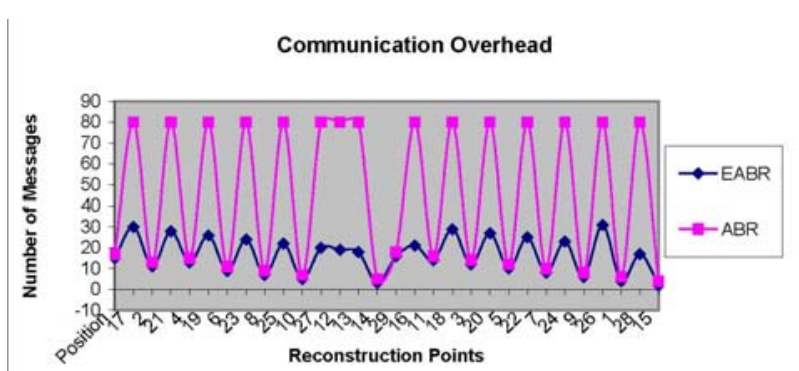

Fig. 1: Communication Overhead: Network $=50$, Route Length $=30$

Corresponding Author: $\quad$ Ayman Mansour Murad, Education Science Faculty - UNRWA, Jordan. P.O Box 140157, Amman 11814 Jordan. Tel: 00962788239237 , Fax: 0096255808289 


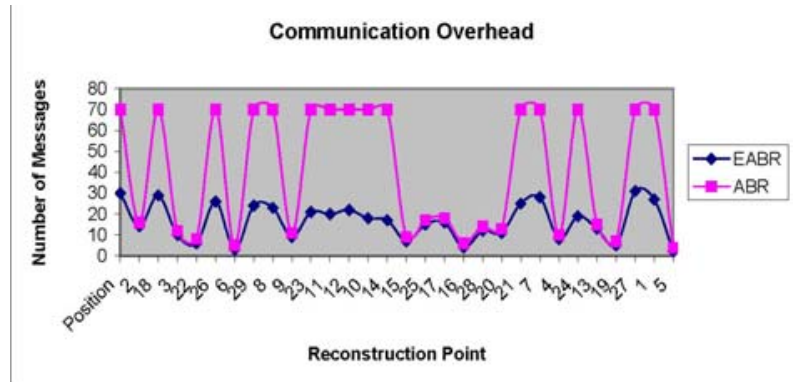

Fig. 2: Communication Overhead: Network $=40$, Route Length $=30$

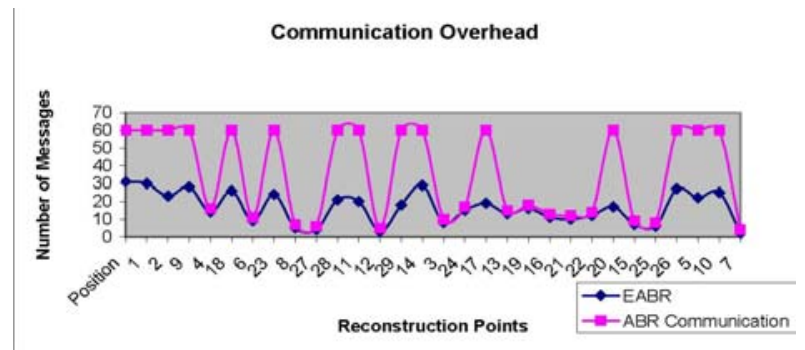

Fig. 3: Communication Overhead: Network $=30$, Route Length $=30$

Network $=\mathbf{3 0}-\mathbf{5 0}$ Nodes - Route $=\mathbf{2 5}$ nodes: In this scenario the number of nodes, which constitute the route, are 25 while the number of nodes constitute the entire network are varying from 30 to 50 . Different results were observed as shown in Figures $4,5 \& 6$.

The simulation results showed that the EABR enhancement in route reconstruction ranges from 34\% to $38 \%$ under different topologies. In cases when the reconstruction is done in the first half of the route the enhancement ranges from $62 \%$ to $72 \%$ under the different topologies, while enhancement reached 12\% when the reconstruction is done in the second half of the route. The communication statistics are summarized in Table 2.

Table 2: Communication Statistics - Route $=25$ nodes under different topologies

Number of Nodes in Network $\begin{array}{lll}50 & 40 \quad 30\end{array}$

EABR Enhancement in Communication EABR Enhancement in Communication in case the reconstruction point fail in the 1st half of the route EABR Enhancement in Communication in case the reconstruction point fail in the 2 nd half of the route

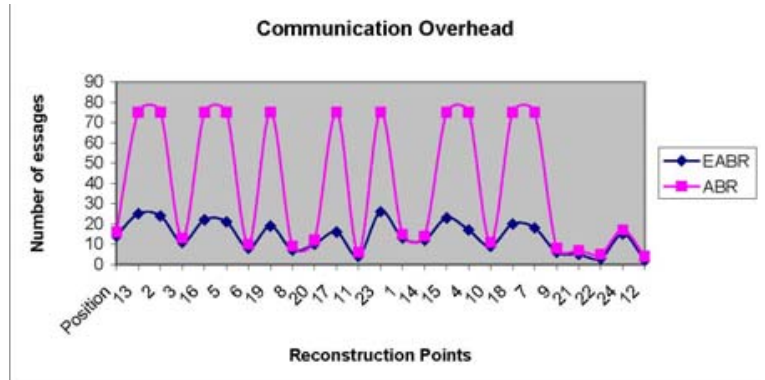

Fig. 4: Communication Overhead: Network $=50$, Route Length $=25$

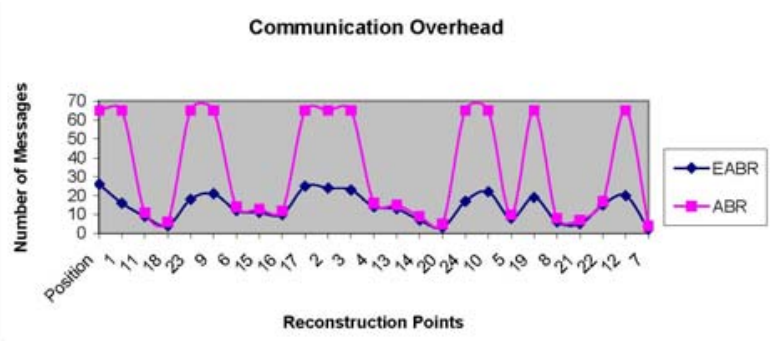

Fig. 5: Communication Overhead: Network $=40$, Route Length $=25$

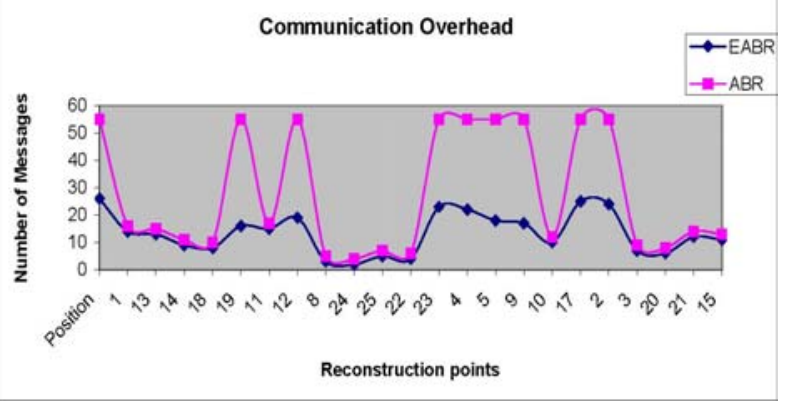

Fig. 6: Communication Overhead: Network $=30$, Route Length $=25$

Network $=\mathbf{3 0}-\mathbf{5 0}$ Nodes - Route $=\mathbf{2 0}$ nodes: In this scenario the number of nodes, which constitute the route, are 20 while the number of nodes constitute the entire network are varying from 30 to 50 . Different results were observed as shown in Figs. 7, 8 \& 9.

The simulation results showed that the EABR enhancement in route reconstruction ranges from 38\% to $42 \%$ under different topologies. In cases when the reconstruction is done in the first half of the route the enhancement ranges from $66 \%$ to $76 \%$ under the different topologies, while enhancement reached $14 \%$ when the reconstruction is done in the second half of the route. The communication statistics are summarized in Table 3. 
Table 3: Communication Statistics - Route $=20$ nodes under different topologies

\section{Number of Nodes in Network}

$\begin{array}{lll}50 & 40 & 30\end{array}$

EABR Enhancement in Communication EABR Enhancement in Communication in case the reconstruction point fail in $42 \% 40 \% 38 \%$ $76 \% 72 \% 66 \%$ the 1st half of the route EABR Enhancement in Communication in case the reconstruction point fail in $\quad 14 \% 14 \% 14 \%$ the 2 nd half of the route

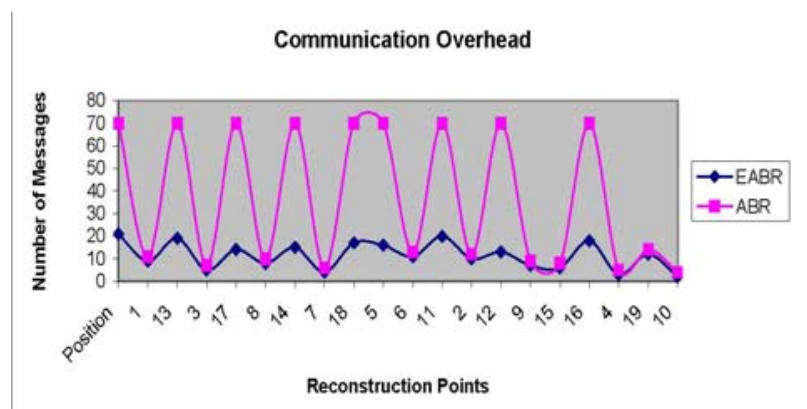

Fig. 7: Communication Overhead: Network $=50$, Route Length $=20$

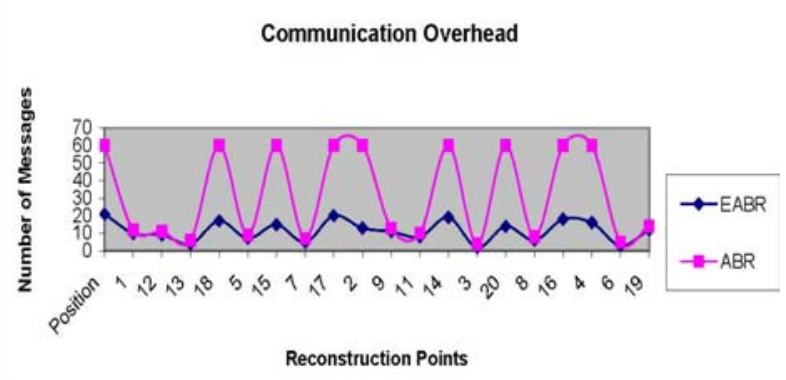

Fig. 8: Communication Overhead: Network $=40$, Route Length $=20$

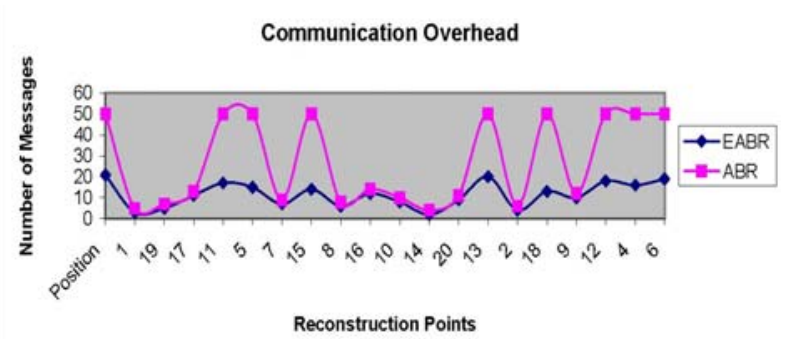

Fig. 9: Communication Overhead: Network $=30$, Route Length $=20$

Network $=30-50$ Nodes - Route $=15$ nodes: In this scenario the number of nodes, which constitute the route, are 15 while the number of nodes constitute the entire network are varying from 30 to 50 . Different results were observed as shown in Figs. 10, 11 \& 12.

The simulation results showed that the EABR enhancement in route reconstruction ranges from 38\% to $47 \%$ under different topologies. In cases when the reconstruction is done in the first half of the route the enhancement ranges from $66 \%$ to $80 \%$ under the different topologies, while enhancement reached $14 \%$ when the reconstruction is done in the second half of the route. The communication statistics are summarized in Table 4.

Table 4: Communication Statistics - Route $=15$ nodes under different topologies

Number of Nodes in Network $\begin{array}{lll}50 & 40 & 30\end{array}$

EABR Enhancement in Communication $47 \% 45 \% 43 \%$ EABR Enhancement in Communication in case the reconstruction point fail in $\quad 80 \% 76 \% 71 \%$ the 1 st half of the route EABR Enhancement in Communication in case the reconstruction point fail in $\quad 18 \% 18 \% 19 \%$ the 2 nd half of the route

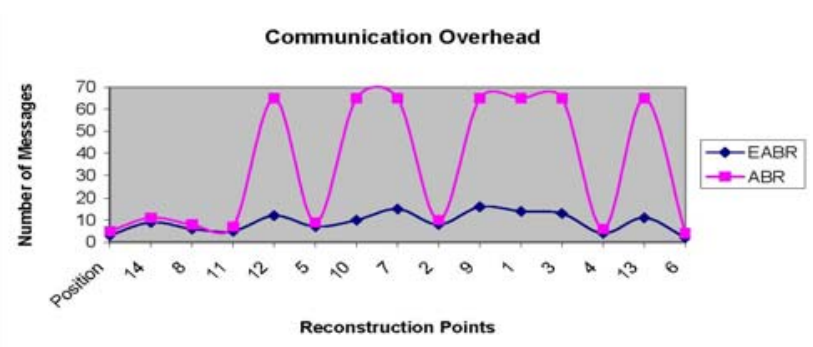

Fig. 10: Communication Overhead: Network $=50$, Route Length $=15$

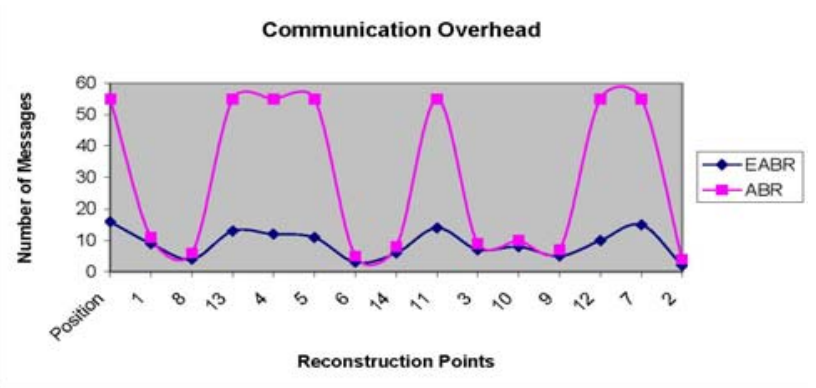

Fig. 11: Communication Overhead: Network $=40$, Route Length $=15$ 


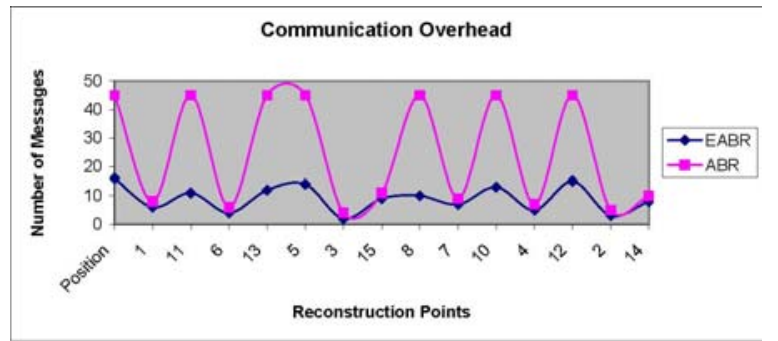

Fig. 12: Communication Overhead: Network $=30$, Route Length $=15$

Network $=\mathbf{3 0}-\mathbf{5 0}$ Nodes - Route $=\mathbf{1 0}$ nodes: In this scenario the number of nodes, which constitute the route, are 10 while the number of nodes constitute the entire network are varying from 30 to 50 . Different results were observed as shown in Figs. 13, 14 \& 15.

The simulation results showed that the EABR enhancement in route reconstruction ranges from $44 \%$ to $47 \%$ under different topologies. In cases when the reconstruction is done in the first half of the route the enhancement ranges from $76 \%$ to $84 \%$ under the different topologies, while enhancement reached $22 \%$ when the reconstruction is done in the second half of the route. The communication statistics are summarized in Table 5.

Table 5: Communication Statistics - Route $=10$ nodes under different topologies

Number of Nodes in Network

$\begin{array}{lll}50 & 40 \quad 30\end{array}$

EABR Enhancement in Communication

$47 \% 46 \% 44 \%$

EABR Enhancement in Communication

in case the reconstruction point fail in

$84 \% 81 \% 76 \%$

the 1st half of the route

EABR Enhancement in Communication

in case the reconstruction point fail in

$22 \% 22 \% 22 \%$

the 2 nd half of the route

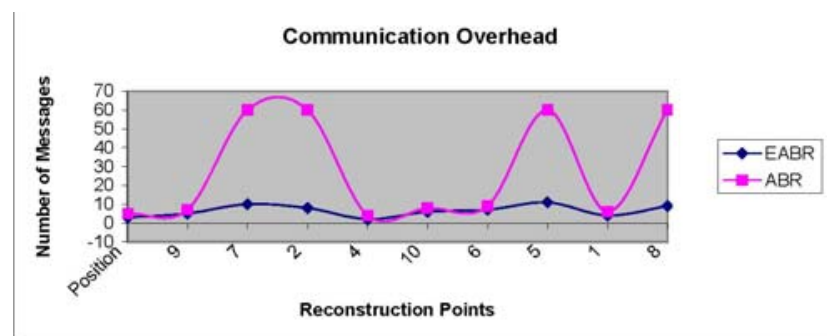

Fig. 13: Communication Overhead: Network $=50$, Route Length $=10$

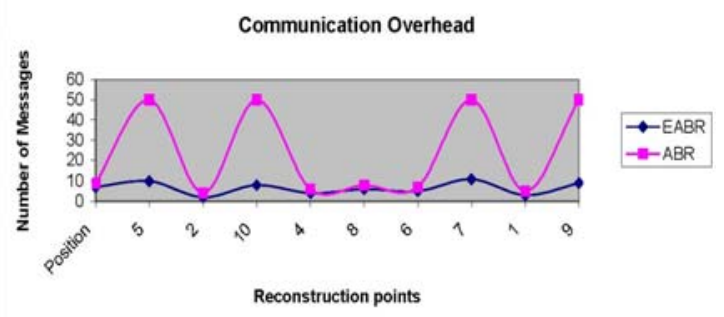

Fig. 14: Communication Overhead: Network $=40$, Route Length $=10$

Communication Overhead

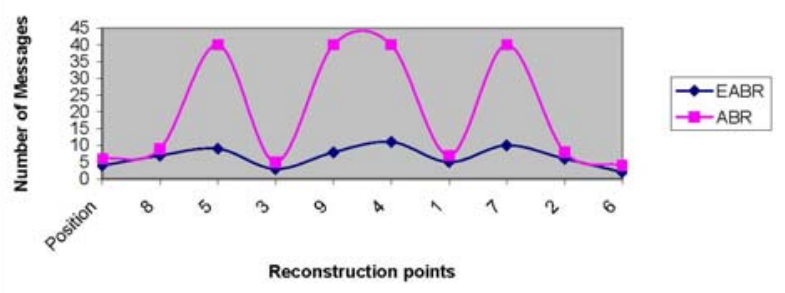

Fig. 15: Communication Overhead: Network $=30$, Route Length $=10$

Network $=\mathbf{3 0}-\mathbf{5 0}$ Nodes - Route $=\mathbf{5}$ nodes: In this scenario the number of nodes, which constitute the route, are 5 while the number of nodes constitute the entire network are varying from 30 to 50 . Different results were observed as shown in Figs. 16, $17 \& 18$.

The simulation results showed that the EABR enhancement in route reconstruction ranges from 54\% to $56 \%$ under different topologies. In cases when the reconstruction is done in the first half of the route the enhancement ranges from $84 \%$ to $90 \%$ under the different topologies, while enhancement reached 33\% when the reconstruction is done in the second half of the route. The communication statistics are summarized in Table 6.

Table 6: Communication Statistics - Route $=5$ nodes under different topologies

Number of Nodes in Network $\begin{array}{lll}50 & 40 & 30\end{array}$

EABR Enhancement in Communication $56 \% 55 \% 54 \%$ EABR Enhancement in Communication in case the reconstruction point fail in $90 \% 88 \% 84 \%$ the 1st half of the route EABR Enhancement in Communication in case the reconstruction point fail in $\quad 33 \% 33 \% 33 \%$ the 2 nd half of the route 


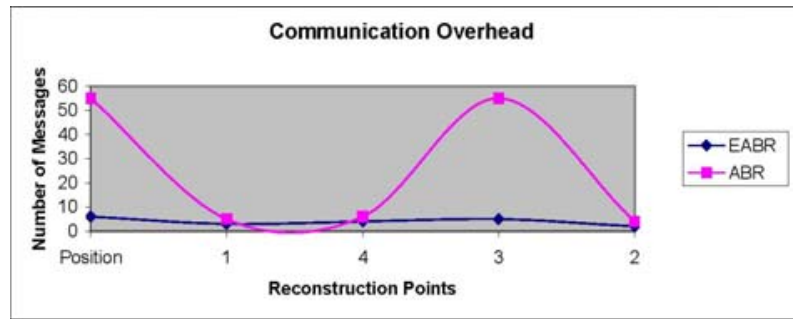

Fig.16: Communication Overhead: Network $=50$, Route Length $=5$

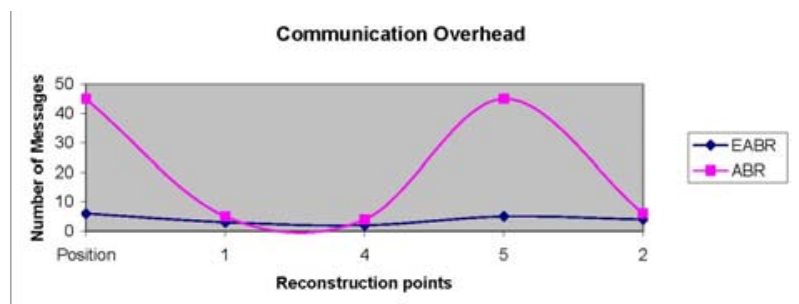

Fig. 17: Communication Overhead: Network $=40$, Route Length $=5$

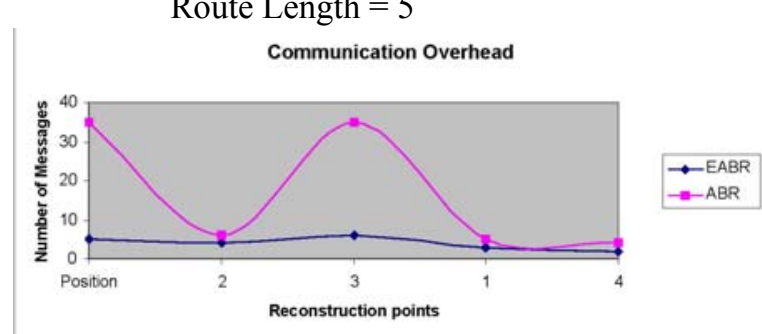

Fig. 18: Communication Overhead: Network $=30$,

Route Length $=5$

\section{Study of Operation Overhead}

Network $=\mathbf{3 0}-\mathbf{5 0}$ Nodes - Route $=\mathbf{3 0}$ nodes: In this scenario the number of nodes, which constitute the route, are 30 while the number of nodes constitute the entire network are varying from 30 to 50 . Different results were observed as shown in Figs. 19, 20 \& 21.

The simulation results showed that the EABR enhancement in route reconstruction reached 36\% under different topologies. In cases when the reconstruction is done in the first half of the route the enhancement reached $66 \%$ under the different topologies, while enhancement reached $11 \%$ when the reconstruction is done in the second half of the route. The operation statistics are summarized in Table
Table 7: Operation Statistics - Route $=30$ nodes under different topologies

Number of Nodes in Network

$50 \quad 40 \quad 30$

EABR Enhancement in Operation $36 \% 36 \% 36 \%$

EABR Enhancement in Operation in case the reconstruction point fail in the $66 \% 66 \% 66 \%$ 1st half of the route

EABR Enhancement in Operation in case the reconstruction point fail in the $\quad 11 \% 11 \% 11 \%$ 2nd half of the route

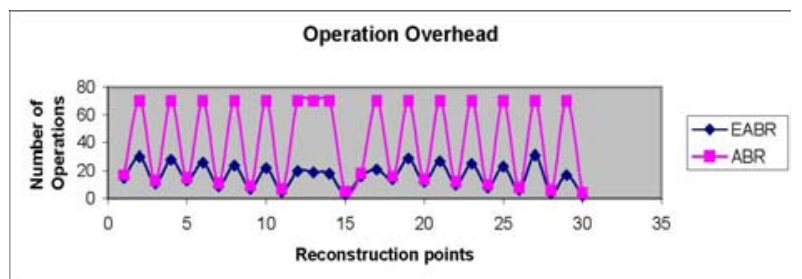

Fig. 19: Operation Overhead: Network $=50$, Route Length $=30$

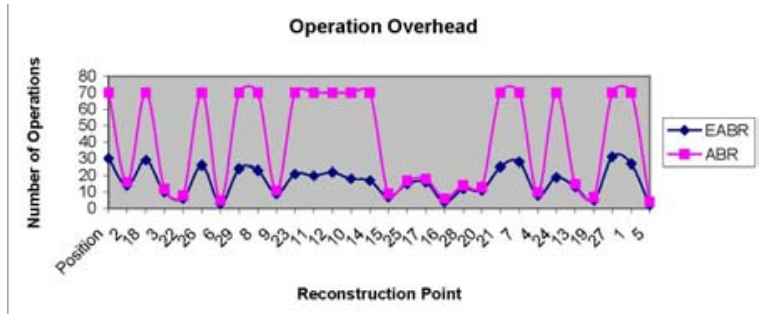

Fig. 20: Operation Overhead: Network $=40$, Route Length $=30$

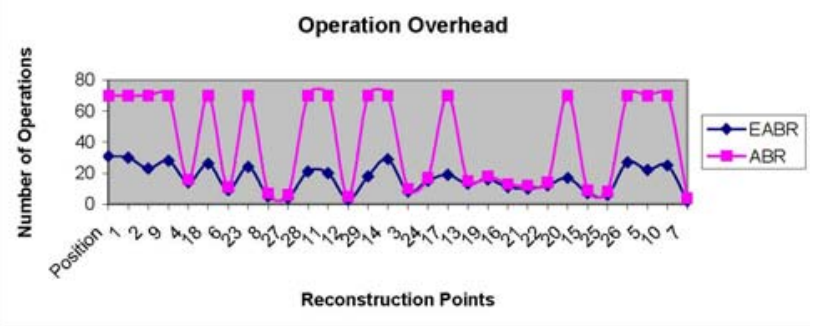

Fig. 21: Operation Overhead: Network $=30$, Route Length $=30$

Network $=30-50$ Nodes - Route $=\mathbf{2 5}$ nodes: In this scenario the number of nodes, which constitute the route, are 25 while the number of nodes constitute the entire network are varying from 30 to 50 . Different results were observed as shown in Figs. 22, 23 \& 24.

The simulation results showed that the EABR enhancement in route reconstruction reached 38\% under different topologies. In cases when the 
reconstruction is done in the first half of the route the enhancement reached $68 \%$ under the different topologies, while enhancement reached $12 \%$ when the reconstruction is done in the second half of the route. The operation statistics are summarized in Table 8 .

Table 8: Operation Statistics - Route $=25$ nodes under different topologies

Number of Nodes in Network $\begin{array}{lll}50 & 40 & 30\end{array}$

EABR Enhancement in Operation $38 \% 38 \% 38 \%$

EABR Enhancement in Operation in case the reconstruction point fail in the 1st half $68 \% 68 \% 68 \%$ of the route EABR Enhancement in Operation in case the reconstruction point fail in the 2nd $12 \% 12 \% 12 \%$ half of the route

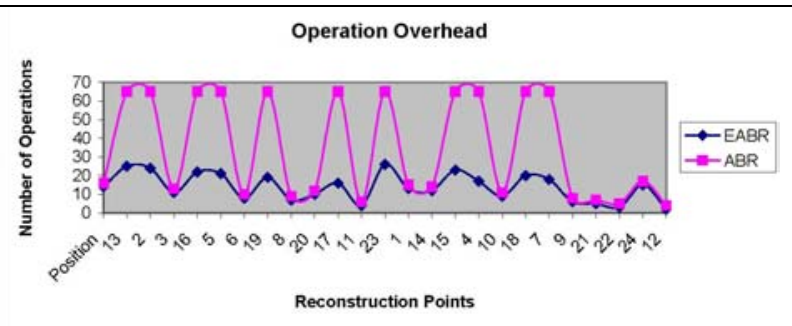

Fig. 22: Operation Overhead: Network $=50$, Route Length $=25$

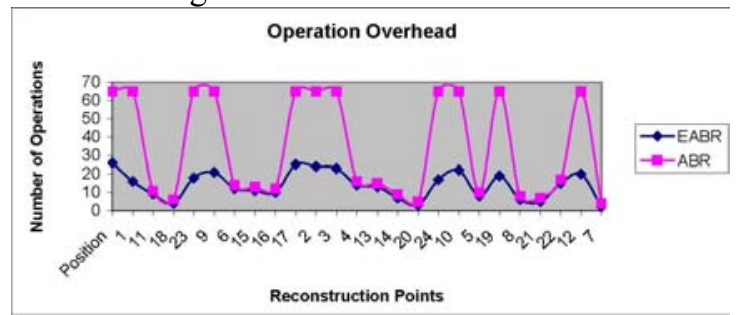

Fig. 23: Operation Overhead: Network $=40$, Route Length $=25$

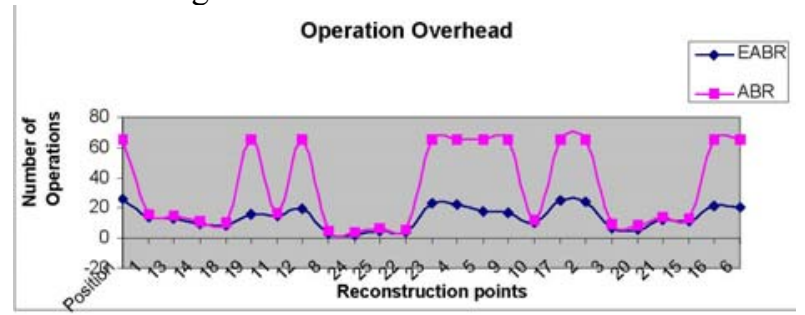

Fig. 24: Operation Overhead: Network $=30$, Route Length $=25$

Network $=\mathbf{3 0}-\mathbf{5 0}$ Nodes - Route $=\mathbf{2 0}$ nodes: In this scenario the number of nodes, which constitute the route, are 20 while the number of nodes constitute the entire network are varying from 30 to 50 . Different results were observed as shown in Figs. 25, 26 \& 27.
The simulation results showed that the EABR enhancement in route reconstruction reached $40 \%$ under different topologies. In cases when the reconstruction is done in the first half of the route the enhancement reached $72 \%$ under the different topologies, while enhancement reached $14 \%$ when the reconstruction is done in the second half of the route. The Operation statistics are summarized in Table 9.

Table 9: Operation Statistics - Route $=20$ nodes under different topologies

Number of Nodes in Network

$\begin{array}{lll}50 & 40 & 30\end{array}$

EABR Enhancement in Operation $40 \% 40 \% 40 \%$

EABR Enhancement in Operation in case the reconstruction point fail in the $72 \% 72 \% 72 \%$ 1 st half of the route

EABR Enhancement in Operation in case the reconstruction point fail in the $\quad 14 \% 14 \% 14 \%$ 2nd half of the route

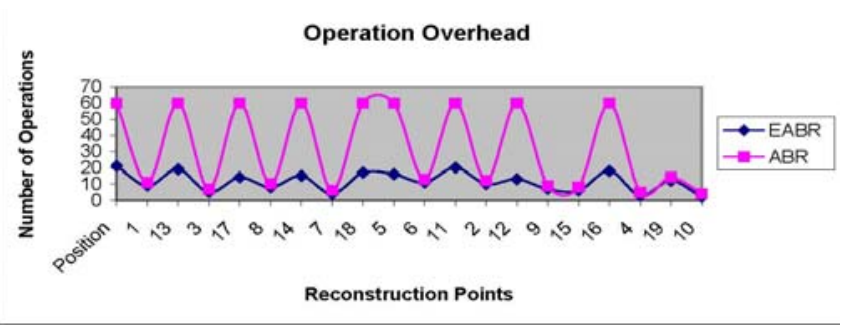

Fig. 25: Operation Overhead: Network $=50$, Route Length $=20$

Operation Overhead

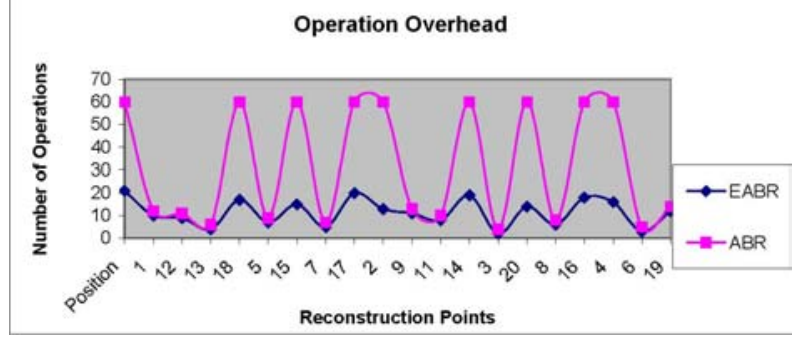

Fig. 26: Operation Overhead: Network $=40$, Route Length $=20$

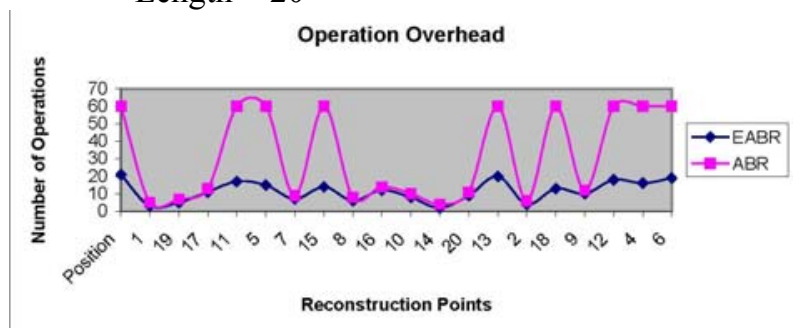

Fig. 27: Operation Overhead: Network $=30$, Route Length $=20$ 
Network $=30-50$ Nodes - Route $=15$ nodes: In this scenario the number of nodes, which constitute the route, are 15 while the number of nodes constitute the entire network are varying from 30 to 50 . Different results were observed as shown in Figs. 28, $29 \& 30$

The simulation results showed that the EABR enhancement in route reconstruction reached $45 \%$ under different topologies. In cases when the reconstruction is done in the first half of the route the enhancement reached $76 \%$ under the different topologies, while enhancement reached $18 \%$ when the reconstruction is done in the second half of the route. The Operation statistics are summarized in Table 10.

Table 10: Operation Statistics - Route $=15$ nodes under different topologies

Number of Nodes in Network $50 \quad 40 \quad 30$

EABR Enhancement in Operation $45 \% 45 \% 45 \%$

EABR Enhancement in Operation in case the reconstruction point fail in the $76 \% 76 \% 76 \%$ 1 st half of the route

EABR Enhancement in Operation in case the reconstruction point fail in the $18 \% 18 \% 18 \%$ 2nd half of the route

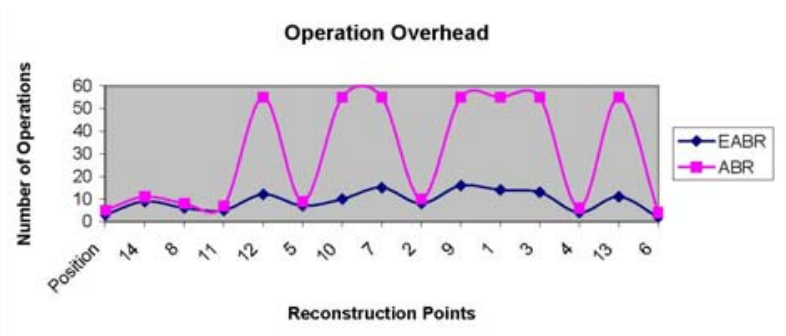

Fig. 28: Operation Overhead: Network $=50$, Route Length $=15$

Operation Overhead

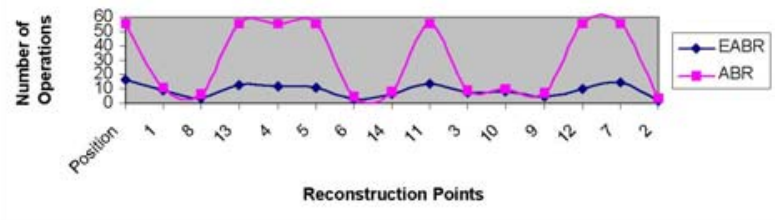

Fig. 29: Operation Overhead: Network $=40$, Route Length $=15$

Operation Overhead

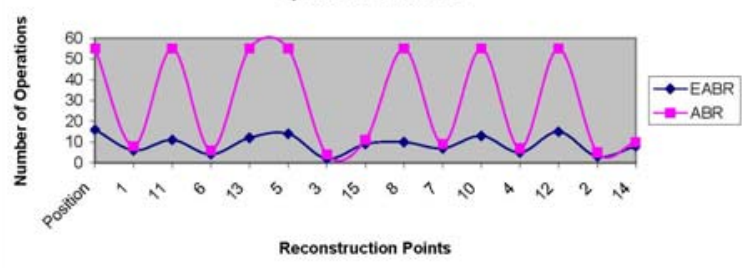

Fig. 30: Operation Overhead: Network $=30$, Route Length $=15$
Network $=30-50$ Nodes - Route $=10$ nodes: In this scenario the number of nodes, which constitute the route, are 10 while the number of nodes constitute the entire network are varying from 30 to 50. Different results were observed as shown in Figs. $31,32 \& 33$.

The simulation results showed that the EABR enhancement in route reconstruction reached $46 \%$ under different topologies. In cases when the reconstruction is done in the first half of the route the enhancement reached $81 \%$ under the different topologies, while enhancement reached $22 \%$ when the reconstruction is done in the second half of the route. The Operation statistics are summarized in Table 11.

Table 11: Operation Statistics - Route $=10$ nodes under different topologies

Number of Nodes in Network $\quad 50 \quad 40 \quad 30$

EABR Enhancement in Operation $\quad 46 \% \quad 46 \% \quad 46 \%$

EABR Enhancement in Operation

in case the reconstruction point $\quad 81 \% \quad 81 \% \quad 81 \%$

fail in the 1 st half of the route

EABR Enhancement in Operation

in case the reconstruction point $\quad 22 \% \quad 22 \% \quad 22 \%$ fail in the 2 nd half of the route

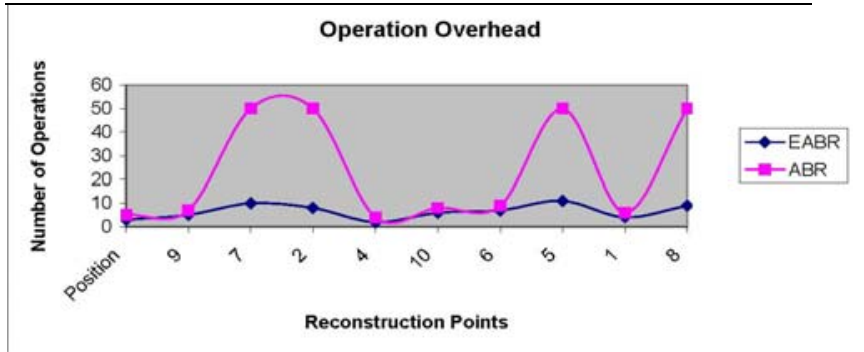

Fig. 31: Operation Overhead: Network $=50$, Route Length $=10$

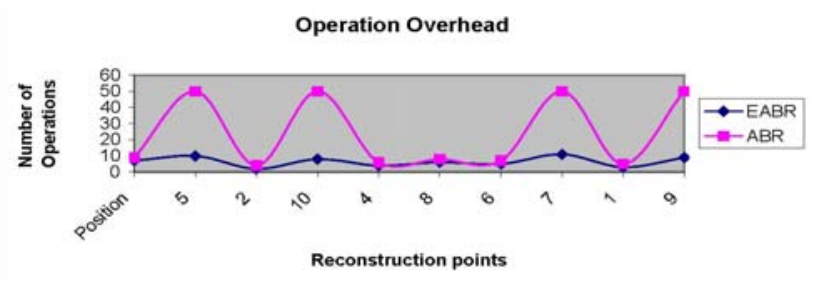

Fig. 32: Operation Overhead: Network $=40$, Route Length $=10$

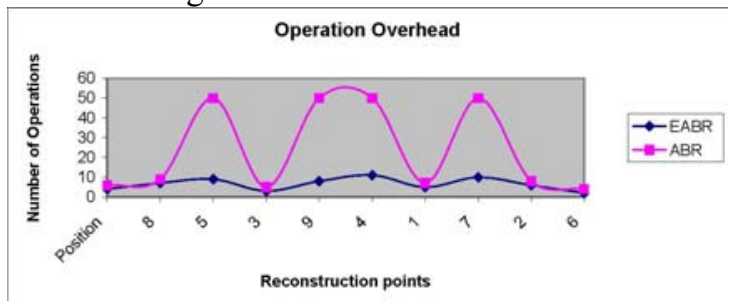

Fig. 33: Operation Overhead: Network $=30$, Route Length $=10$ 
Network $=30-50$ Nodes - Route $=5$ nodes: In this scenario the number of nodes, which constitute the route, are 5 while the number of nodes constitute the entire network are varying from 30 to 50 . Different results were observed as shown in Figs. 34, 35 \& 36.

The simulation results showed that the EABR enhancement in route reconstruction reached 55\% under different topologies. In cases when the reconstruction is done in the first half of the route the enhancement reached $88 \%$ under the different topologies, while enhancement reached $33 \%$ when the reconstruction is done in the second half of the route. The Operation statistics are summarized in Table 12.

Table 12: Operation Statistics - Route $=5$ nodes under different topologies

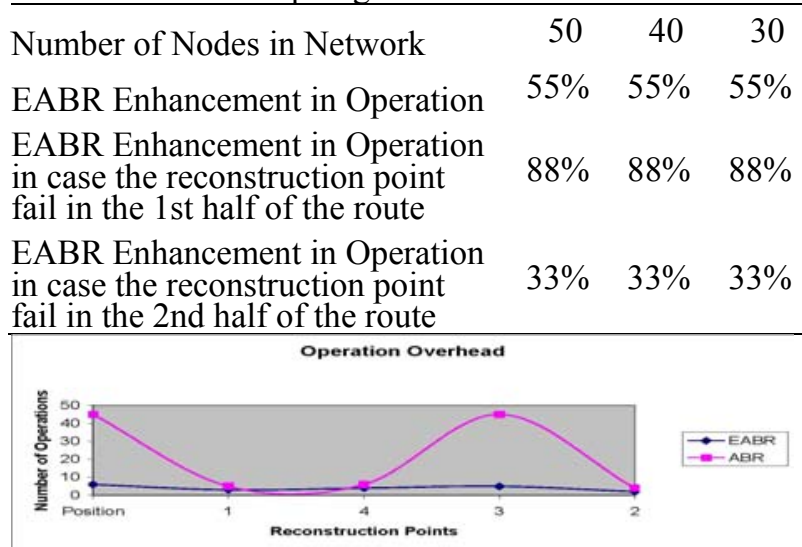

Fig. 34: Operation Overhead: Network $=50$, Route Length $=5$

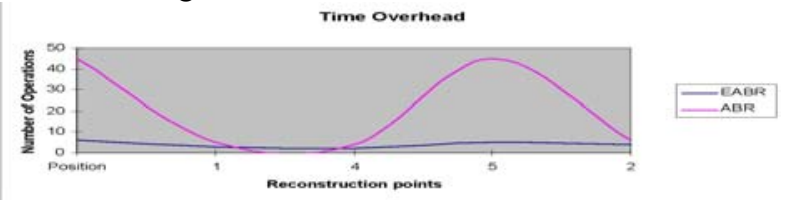

Fig. 35: Operation Overhead: Network $=40$, Route Length $=5$

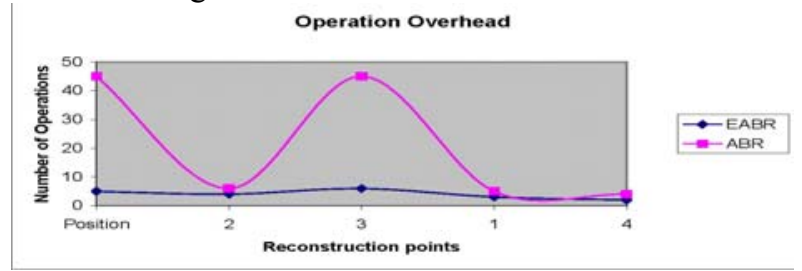

Figure 36: Operation Overhead: Network $=30$, Route Length $=5$

\section{RESULTS AND DISCUSSION}

The simulation study aimed to study the performance the EABR and original the ABR protocols in route reconstruction phase. The two factors used to conduct the simulation analysis between the protocols are: Operation Complexity and Communication Complexity as defined in ${ }^{[1]}$.

The study was restricted by the following assumptions in order to study the performance in route reconstruction phase only, no other cases were included in the study as it fails beyond the scope of our interest.

1. Operation complexity ${ }^{[1]}$ and Communication

2. Ideal environment i.e. no interference or noise signals

3. A conference-sized network ranging from 30 to 50 nodes

4. 2 nodes can communicate/ respond with each other if they are in the transmission range

5. Nodes move in random manner

6. The route is already established

The simulation strategy was based on measuring the communication overhead in terms of number of messages exchanged to perform a routing operation, and the Operation overhead in terms of the number of operations required to perform a protocol operation as defined in ${ }^{[1]}$.

Different topologies ranging from 30 to 50 nodes were used; also different route lengths ranging from 5 to 30 nodes were used in this simulation study.

\section{CONCLUSION}

1. The overall EABR enhancement in terms of communication overhead is ranging from $36 \%$ to $56 \%$.

2. The overall EABR enhancement in terms of number of operation required to reconstruct the route is ranging from $36 \%$ to $55 \%$.

3. The EABR communication enhancement in case the reconstruction point fail in the 1st half of the route is ranging from $60 \%$ to $90 \%$.

4. The EABR communication enhancement in case the reconstruction point fail in the 2 nd half of the route is ranging from $11 \%$ to $33 \%$.

5. The EABR Operation enhancement in case the reconstruction point fail in the 1 st half of the route is ranging from $66 \%$ to $88 \%$.

6. The EABR Operation enhancement in case the reconstruction point fail in the 2 nd half of the route is ranging from $11 \%$ to $33 \%$.

7. In the backtracking process of the ABR, the process stops after reaching the node, which represent half of the hop count, which can be optimized more as there is a big possibility to have the destination to settle near to the upper part of the route.

8. When the network consists of relatively a large number of nodes and the established route consists of relatively small number of routes, the ABR is not efficient in route reconstruction, while the EABR is better in such topologies if compared to the ABR.

\section{REFERENCES}

1. C.-K. Toh, 2002. Ad Hoc Mobile Wireless Networks: Protocols and Systems, Prentice Hall PTR, Prentice Hall, Inc. PP 79-116

2. Ayman, M., Said A. 2006. Enhanced Routing ReConstruction Method for the Associativity Based Routing Protocol for Mobile AD Hoc Network (MANET)", American Journal of Applied Sciences. PP 859-869

3. Ayman, M., Said A. 2006. Analysis of Enhanced Associativity Based Routing Protocol", American Journal of Applied Sciences. PP 853-858 